$\Delta$ Palabras clave/ Arquitectura, paisaje, imitación, proyecto.

$\Delta$ Keywords/ Architecture, landscape, imitation, project.

$\Delta$ Recepción/ 12 junio 2018

$\Delta$ Aceptación/ 13 agosto 2018

\title{
La imitación del paisaje en el proyecto arquitectónico contemporáneo
}

\author{
The imitation of landscape in \\ contemporaneous architectural projects
}

\section{Santiago Quesada-García}

Arquitecto, Universidad de Sevilla, España

Doctor en Arquitectura, Universidad de Sevilla España.

Profesor, Departamento de Proyectos

Arquitectónicos, Universidad de Sevilla, España.

sqg@us.es

RESUMEN/ El paisaje se configura, hoy en día, como una estructura de relaciones que el proyecto de arquitectura reconstruye, definiendo un marco de pertenencia histórico, cultural, ético y estético en el cual se enmarca y adquiere sentido e identidad el propio proyecto. El actual concepto de paisaje no posee la rigidez, estaticidad y perfección de la naturaleza pre-moderna cuando era uno de los modelos de imitación a seguir según el postulado aristotélico Ars imitatio naturae. La tesis de este artículo es que el proyecto arquitectónico es una acción o praxis que adquiere su principal motivación en un deseo de imitación racional, consciente y libre de determinados prototipos o referentes. Uno de ellos es el paisaje. El paisaje se configura en la actualidad como un referente analógico, emocional o figurativo para la arquitectura. En definitiva, es uno de los modelos de imitación utilizados durante el proceso de proyecto contemporáneo. ABSTRACT/ At present, landscapes are configured as an array of relationships reconstructed by the architectural project. A historical, cultural, ethical and aesthetical ownership framework is defined, which frames the project itself, granting it meaning and identity. The current concept of landscape is not as rigid, static and perfected as the pre-modern nature, when it was one of the imitation models to be followed under the Aristotelian tenet: ars imitatio naturae. This work suggests that the architectural project is an action or praxis basically motivated by a rational, conscious, and free imitation desire of certain prototypes or models. One of these models is landscape. Currently, landscape emerges as an analogical, emotional or figurative model for architecture. Ultimately, it is one of the imitation models used during the contemporaneous project process.

La antigua relación arquitectura-naturaleza tiene su ratificación contemporánea en la idea de paisaje que es considerada hoy por la arquitectura como un ideal a alcanzar en sus realizaciones. El actual concepto de paisaje supera la vieja distinción entre fondo y figura, entre objeto y sujeto. En la ambigüedad y polisemia del término 'paisaje' se reconocen en el presente los límites entre el mundo físico y fenomenológico que separó la visión ilustrada del siglo XVIII. De esta forma la noción contemporánea de paisaje representa una superación entre forma y contenido; Es un lugar o límite en el que se sobreponen imagen y realidad.

A través de la praxis del proyecto -y no de la teoría o de la abstracción conceptual-, la arquitectura redescubre el paisaje como fundamento y modelo del proyecto. Para justificar esta afirmación es necesario establecer en primer lugar qué se entiende por paisaje en la disciplina arquitectónica; Posteriormente, definir en qué consiste la acción imitativa cuando se realiza un proyecto; Y, por último, establecer por qué se elige el paisaje como modelo. Estas serán las secciones argumentales a través de las cuales discurrirá y se desarrollará este artículo, las cuales son precedidas por una breve contextualización temática que se expone a continuación. 
El paisaje, en la cultura occidental, ha sido una creación de la pintura. Su reciente puesta en valor proviene de la ecología, de la geografía y de la antropología, pero, sobre todo, de la fenomenología. No obstante, casi todas las interpretaciones del paisaje se han enmarcado en un contexto filosófico en el que el llamado 'paradigma lingüístico' ha tenido un predominio incontestable. Según este paradigma, la realidad se estructura lingüísticamente y solo se conoce por medio del lenguaje natural o corriente, que es una creación social e histórica'. El geógrafo francés Augustin Berque sostiene que el paisaje es un concepto que no existía en la cultura occidental sino hasta la aparición en el siglo XVII de un término capaz de describir lingüísticamente el significado de dicho concepto. Este fuerte predominio del lenguaje referido al concepto de paisaje ha dificultado la verdadera comprensión de su naturaleza, porque ha valorado en exceso su componente visual, ha ignorado cuál es la experiencia fenomenológica que se puede extraer del mismo y cuál es el papel que puede desempeñar como modelo o referencia para los arquitectos ${ }^{2}$. A ello hay que unir que, a comienzos del siglo veinte, la máquina se convirtió en la expresión de una nueva cultura; era el mito salvador a lo que todo se debía someter, incluso la creación artística.

La superioridad técnica de la máquina acabó desplazando a la naturaleza, considerándola como algo externo y extraño al hombre y convirtiéndola en un elemento que debía ser conservado y mantenido. Se reservaron numerosos espacios naturales para convertirlos en parques o zonas con una protección de raíz esencialmente ambientalista, que buscaba la preservación de esas áreas con un componente natural muy marcado y una
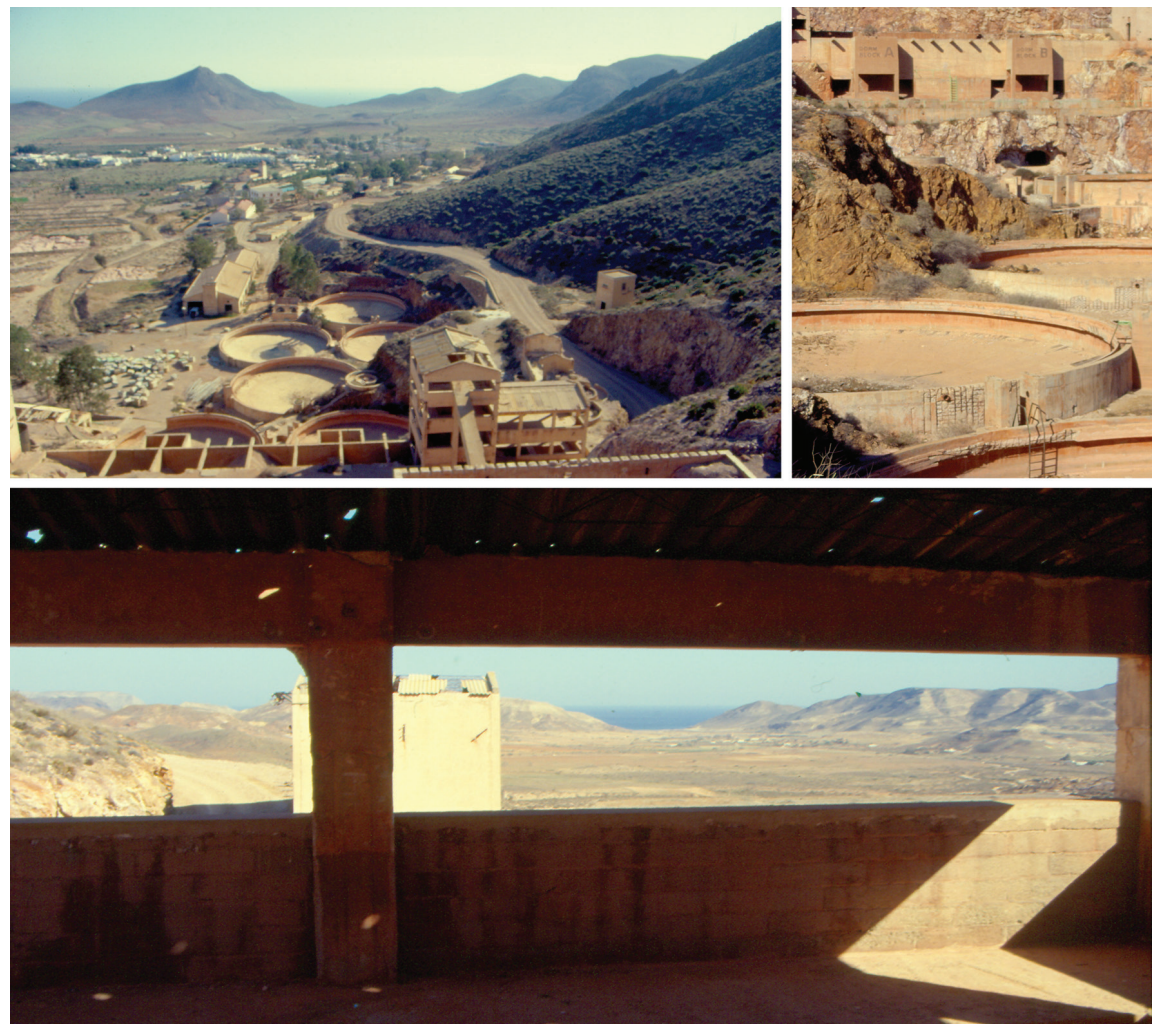

Imagen 1. Restos de la factoría de beneficio de oro construida en Rodalquilar (España) y ahora integrados en el Parque Natural del Cabo de Gata en Almería (fuente: El autor 1998).

escasa presencia humana. Protección que, a la vez, ignoraba la inevitable antropización realizada por el hombre a lo largo de los siglos. Esta sobrevaloración de lo natural ha desembocado en una especie de 'frenesí eco-paisajístico', que ha incitado movimientos masivos de población para el disfrute y contemplación de determinados panoramas que, en algunos casos, se denominan 'paisajes'. Se va al encuentro de la naturaleza y se es espectador de una obra producida por el propio hombre.
Transcurrido un siglo, aquella ilusión por la máquina ha sido sustituida por la fascinación que ejercen sus propias ruinas convertidas en paisaje, como demuestran los numerosos restos post-industriales ahora integrados y conservados en parques naturales o ciudades de América y Europa. Algunos ejemplos de estas intervenciones son el High Line en Nueva York, el Landschaft Park en la cuenca del Ruhr o la factoría de oro en el Parque Natural Cabo de Gata en Rodalquilar (Quesada-García 2000).

${ }^{1}$ El paradigma lingüistico implica un universal abstracto en el que los nombres comunes del lenguaje hacen referencia a una esencia ideal y genérica, nunca a sustancias individuales y concretas 


\section{EL CONCEPTO DE PAISAJE EN LA DISCIPLINA ARQUITECTÓNICA.}

Analizando con una nueva mirada la obra de algunos arquitectos de las vanguardias del siglo XX, particularmente atentos al tema de la relación hombre-naturaleza, se redescubre la raíz moderna del paisaje como material de proyecto. Durante sus años de aprendizaje, los arquitectos de las vanguardias perciben el paisaje todavía desde un punto de vista pictórico. Un claro ejemplo son los dibujos de Le Corbusier durante su viaje por la Toscana, cuando era estudiante, en los que la arquitectura del Duomo de Florencia se disuelve en las brumas del ambiente. Más tarde, en pleno ejercicio profesional, estos pioneros modernos demuestran su sensibilidad hacia el paisaje cuando introducen sus obras arquitectónicas como objetos o figuras en un paisaje que actúa como fondo, considerando la relación entre objeto y sujeto todavía en sentido clásico. Sin embargo, se descubre su sensibilidad hacia el paisaje a través de varios acercamientos indirectos. El primero de ellos, ligado a la reelaboración de conceptos de la arquitectura occidental, se identifica en las obras de Le Corbusier y Mies van der Rohe. El arquitecto franco-suizo demuestra su atención al evocador poder del paisaje cuando coloca sus edificios con el paisaje como fondo y este es contemplado desde dentro, por medio de la promenade architecturale, como si fuera un cuadro de la propia casa. El arquitecto alemán lo hace por medio del uso de una

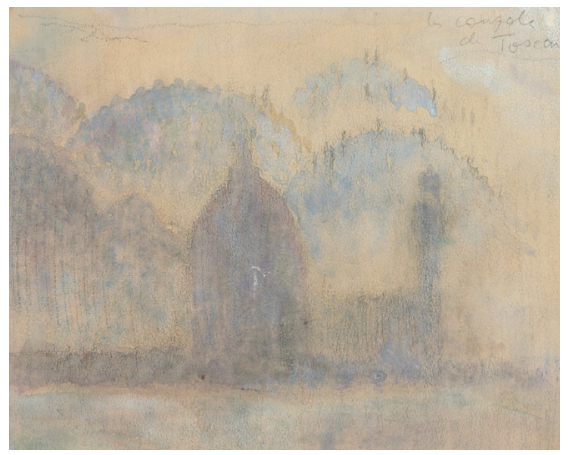
Imagen 2. Vista de la cúpula del Duomo Santa Maria del Fiore
y Palazzo Vecchio, Florencia, 1907, Le Corbusier. Dibujo de viaje por la Toscana (fuente: AA.V. . 1987). estética en la que lo natural y lo artificial se funden en un solo elemento, como ocurre en el ejemplo de la casa Farnsworth. Otra aproximación moderna al paisaje deriva de la arquitectura oriental y, en particular, de la tradición doméstica japonesa, de la dimensión íntima de su espacio interior y la importancia del jardín. Ejemplo de esta sensibilidad son las obras americanas de Rudolph Schindler y Richard Neutra. Una tercera modalidad de acercamiento al paisaje por la arquitectura moderna viene de la mano de la fascinación por la tradición de la arquitectura mediterránea con sus patios y jardines interiores, sensibilidad que estaría representada por la obra del mexicano Luis Barragán.

A esas aproximaciones indirectas al paisaje, enunciadas por el crítico Kenneth Frampton (1991), habría que añadir el acercamiento propuesto por Dimitris Pikionis. Este arquitecto, en su intervención en los caminos y accesos de la Acrópolis, diluye por completo, por vez primera, el límite entre objeto y sujeto. El arquitecto griego diseña un nuevo paisaje compuesto por piedras que en sí son ya arquitectura. Reconstruye un paisaje mítico perdido que recompone, como por encanto, fragmento por fragmento, en una obra en la que paisaje y arquitectura se convierten en un todo indisoluble.

Todos estos arquitectos modernos, con su una semilla que acabaría fructificando iniciada la década de los setenta del pasado nueva forma de mirar y proyectar, plantaron

siglo, cuando se utiliza conscientemente el paisaje como material de proyecto. Esto sucede cuando los arquitectos Gabetti e Isola construyen el 'Residencial Oeste' en Ivrea, un edificio colocado en las ondulaciones del terreno en torno a una colina verde, cuyo aspecto y percepción cambia con las estaciones del año. Los italianos proponen en este proyecto un retorno a la naturaleza que como ellos mismos dicen "...es imposible, pero existe y todavía una vez más, lo experimentamos" (Pedio 1973: 83). La motivación principal de este proyecto es el deseo de crear un nuevo paisaje con una nueva arquitectura.

La propuesta de Gabetti e Isola consiste en una arquitectura que no se impone al territorio, que no se deposita, sino que parece germinar de él. Crean un nuevo paisaje con una nueva presencia, con una arquitectura que es llevada a una fusión entre figura-fondo, diluyendo así los límites entre objeto y sujeto. Una disolución característica fundamental de la visión contemporánea. La profunda significación que toma en este proyecto el concepto de paisaje hace que en la nueva obra arquitectónica creada en el valle de Aosta se despliegue toda la comprensión y verdad de la idea contemporánea de paisaje con mayor plenitud que en la enunciación lingüística o abstracta de cualquier regla o teoría. E nuevo paisaje construido es un ejemplo, un elemento vivo en continua evolución y cambio y, por tanto, se convierte en un sujeto dinámico y moderno susceptible de proyectarse al futuro.
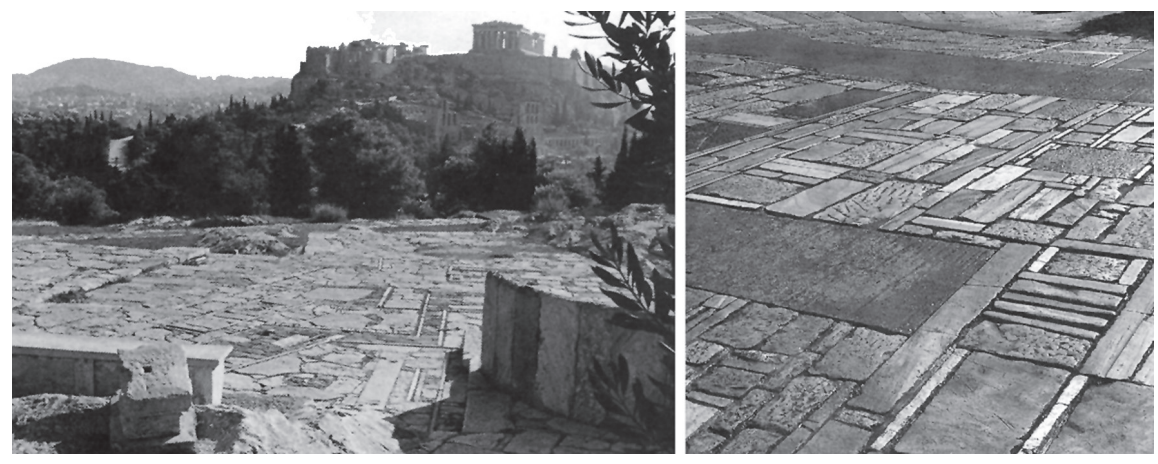

Imagen 3. Senderos de la Acrópolis construidos en 1958 por Dimitri Pikionis. Atenas (Grecia). El arquitecto griego construye un nuevo paisaje con una arquitectura hecha de piedras (fuente: El autor 2003). 
"La mutación es la única característica inmutable del paisaje",3 dice F. L. Wright (Kaufmann 1962). El paisaje siempre está en incesante y constante transformación. El paisaje cambia siempre porque, como la cultura de un pueblo, está también en constante evolución, y como ocurre con la historia de una cultura, de la misma forma puede tener connotaciones positivas o negativas. En el paisaje se encuentran multitud de buenos y malos ejemplos: los bosques de Sherwood, Yosemite o el Dorado, por nombrar algunos. El paisaje, a diferencia de la naturaleza pre-moderna, es un modelo inacabado e imperfecto, carece de su estatismo y finitud. John Brinckerhoff Jackson mantiene que los valores presentes en un paisaje son el espejo de una comunidad, consecuencia del marco histórico, social y cultural que lo ha generado. Por tanto, es un depósito de mitos, fruto de la memoria personal y colectiva, cambiante y mutable, con una sorprendente resistencia a lo largo del tiempo y con capacidad de dar forma a instituciones con las que todavía hoy en día se convive (Jackson 2010). El geógrafo Peter Howard (2014) dice que ante el paisaje -como significado, como imagen, como escala, como escenario y como lugar- las personas pueden sentir emociones profundas y poderosas que solo se asimilan lentamente a lo largo del tiempo. El paisaje se presenta pues como una referencia universal cuya verdad se manifiesta por medio de la experiencia a través de determinadas acciones capaces de producir emoción.

Para la sociedad actual y, por tanto, para la arquitectura contemporánea, el paisaje representa una imperfecta armonía de valores estéticos, económicos, afectivos, emocionales y culturales, que provoca un deseo incoativo y racional de aprehenderlo y experimentarlo por parte de los sujetos que lo perciben. El actual concepto de paisaje puede configurarse a través de la imagen y de la experiencia pero, además, puede ser reconstruido físicamente a través del proyecto arquitectónico. Lo que es significativo en el paisaje es que no es solo una práctica formal o técnica, sino que es capaz de construir experiencias alternativas de ser y de relacionarse. Esto es así porque es capaz de significar a un objeto por medio de su imagen, otorgándole sentido mediante un conjunto de signos y, a la vez, es capaz de ser representación de ese mismo objeto, tal y como mantiene Francisco Ayala (1996). Estas características llevan a concluir que el paisaje no es un objeto inerte, inmutable, perfecto y acabado sino un sujeto dinámico en evolución constante que, más que ser contado, debe ser experimentado, tal y como sucede con la vivencia de la arquitectura. De aquí la afinidad que la disciplina arquitectónica encuentra actualmente con el paisaje y que ha llevado al desarrollo de nuevas disciplinas como la arquitectura del paisaje, en las que este se convierte en uno de los principales materiales del proyecto con el objetivo de diseñar la conformación física de las relaciones de las personas con su contexto ambiental. Una disciplina que ha surgido por el sentimiento de urgencia que prescribe la ecología y por la atención al medio ambiente que demanda la sociedad actual. Ese interés, combinado con el surgimiento de sitios post-industriales obsoletos que exigen técnicas paisajísticas innovadoras, ha provocado el desarrollo de un campo profesional que está definiendo competencias propias e inesperados campos de investigación.

Sin embargo, a pesar de esta nueva especialización surgida en los últimos años, la arquitectura continúa buscando cómo producir obras que enmarquen y provoquen la experiencia que induce el paisaje. De aquí que se indague en los valores y características emocionales del propio paisaje como un modelo a seguir. Para entender cómo el paisaje puede ser un referente de la arquitectura contemporánea es necesario establecer qué se entiende por acción imitativa del proyecto arquitectónico y cómo esta aparece en el mismo.

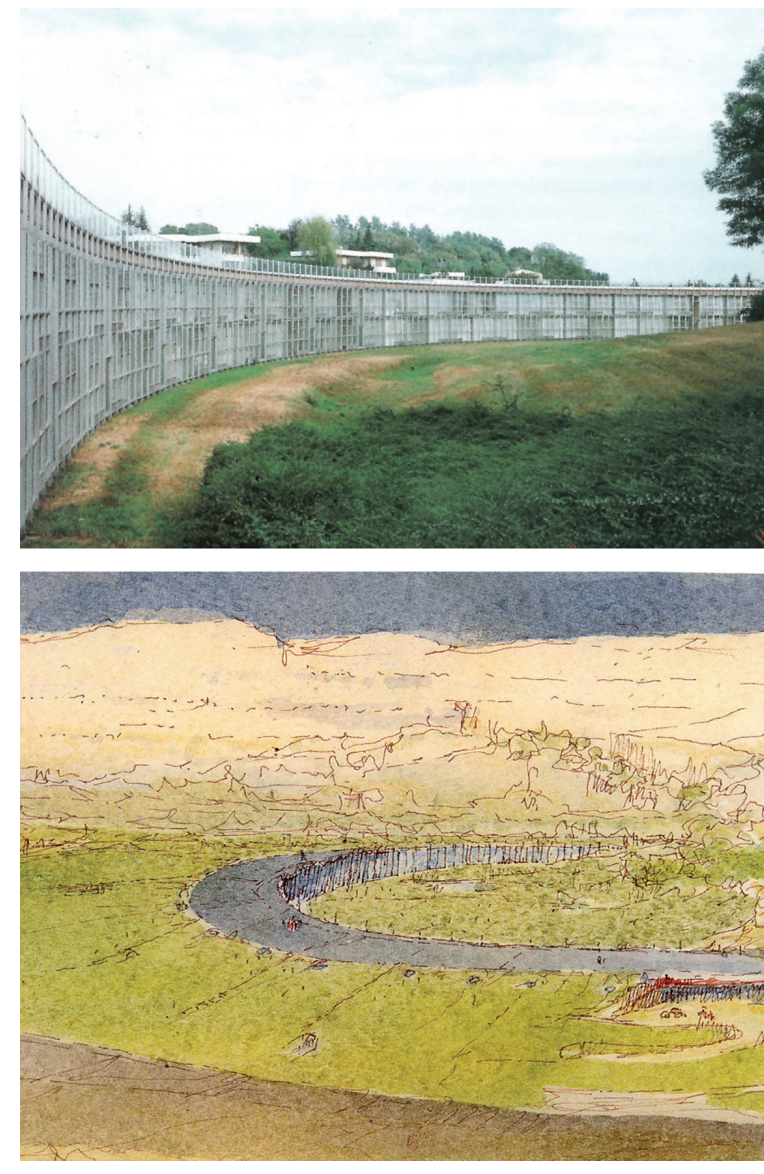

Imagen 4. Gabetti \& Isola con L. Re, Residencial Oeste en Ivrea (Italia), 1968-1971 (fuente: Gabetti e Isola studio en Guerra y Morresi 1996)

3 Si la regla que ilustra el ejemplo es práctica y no térica o técnica, el ejemplo demuestra que un cierto hecho es válido. El ejemplo es un elemento extraño a la abstracción, remite a la concreción. Desde
una perspectiva lógico-conceptual, es impensable que algo concreto sea al mismo tiempo común a todas las cosas: por ello, según ese punto de vista, lo concreto no puede ser nunca universal. El paisaje una perspectiva lógico-conceptual, es impensable que algo concreto sea al mismo tiempo común a todas las cosas; por ello, según ese punto de vista, lo concreto no puede ser nunca universal. El paisaje no se experimenta mirando un cuadro o leyendo una página de literatura. La experiencia emocional que suscita un paisaje se 


\section{LA ACCIÓN IMITATIVA EN EL PROYECTO ARQUITECTÓNICO.}

\section{"Quién sigue a los demás nunca los} adelanta", decía el arquitecto barroco Borromini (1993) parafraseando a Miguel Ángel. Esta frase expresa con claridad la necesidad de superar al modelo elegido, reinterpretándolo a través de nuevos códigos arquitectónicos. La imitación surge cuando se transcribe en clave contemporánea un lenguaje o código heredado debido a la necesidad provocada por la evolución del hombre y de la sociedad a la que pertenece. Esa acción imitativa se hace partiendo de modelos existentes que se revisitan y se eligen de manera consciente, racional y libre. Existe un nuevo tipo de imitación contemporánea que comenzó a nacer a finales del siglo XIX con el pensamiento de Mallarmé, Collodi o Wilde, y que ha sido recientemente teorizada por el filósofo Javier Gomá4. Este pensador mantiene que la imitación contemporánea es un nuevo tipo que supera las pre-modernas basadas en la imitación de los Antiguos, de la Naturaleza y de las Ideas. En esta novedosa mímesis, el modelo elegido para imitar es siempre un sujeto, nunca un objeto. Un sujeto capaz de inspirar que sea imitado por otro sujeto, una tarea que se hace de una manera racional, consciente y libre. Esto implica que la relación imitativa se establece entre dos sujetos diferentes, no entre un objeto y un sujeto, como sucedía en la antigüedad. Esta nueva mímesis contemporánea es la que se produce entre el proyecto arquitectónico y el paisaje.

El proceso proyectual del arquitecto es una acción subjetiva orientada a conseguir un determinado grado de precisión, rigor y coherencia formal en una obra de arquitectura dentro de un sistema estético determinado, en función del marco histórico y cultural en el que se desarrolla. Esa consistencia o identidad formal depende de la materia prima utilizada durante el proceso de proyecto, de manera que ese material es el que permite la construcción de un orden nuevo a partir de elementos, modelos o referentes verificados en el tiempo por medio de la experiencia. Esta praxis, que se realiza durante el proyecto arquitectónico, se puede considerar una acción imitativa. Pero esta imitación no es nunca una simple repetición, con connotaciones reductivas o negativas. La mímesis no es sinónimo de copia o plagio. Al imitar un modelo anhelado o deseado, el producto resultante se ancla en una tradición (una innovación conseguida) y crea algo completamente diferente que se proyecta al futuro. La imitación está en la propia naturaleza del hombre y este, cuando quiere avanzar, la asume de una manera ética y moral. Para los arquitectos, emular un determinado prototipo o modelo no significa ceder al instinto o al impulso de una pasión reproduciendo literalmente lo que ven, sino descubrir el fundamento del modelo elegido para imitar, alcanzando sus valores, perfectos o imperfectos, no repitiendo su imagen, forma o representación, ya que el concepto de imitación moderno supera cualquier mímesis literal de formas preexistentes.

Estando expuesto cotidianamente a la influencia de multitud de ejemplos, prototipos o referentes, la labor del arquitecto será encontrar, entre la pluralidad de lo dado, un modelo normativo digno de elección; un ejemplo válido, que justifique su reiteración en el proyecto. Para ello es necesario investigar a fondo tanto al modelo como la racionalidad en la elección de ese prototipo. Los arquitectos, al proyectar, usan la razón, para reconocer a qué modelo referirse, conocer intimamente mediante la acción de proyectar las leyes profundas del modelo elegido y, finalmente, para transmitir ese conocimiento por medio de las obras realizadas, que no son nunca producto de la invención o ideación ex novo

Por tanto, el proceso de proyecto arquitectónico no es autorreferente sino que siempre alude a modelos, prototipos o referentes que son elegidos de forma libre, consciente y racional durante una praxis de la que siempre se obtiene una enseñanza. Este proceso se trata de una acción que el arquitecto no realiza una sola vez, en un momento aislado, sino a lo largo de la pluralidad de proyectos que se acumulan en el curso de su práctica profesional y que, con los años, proporcionan una determinada experiencia.
Con la ejecución del proyecto y el paso del tiempo, el arquitecto experimenta, con una conciencia cada vez más nítida, la distancia que lo separa de aquel modelo o modelos que eligió como referente y que, a su pesar, nunca alcanza. En el ejemplo que deja cada proyecto terminado se aprecia la diferencia que lo aleja del prototipo elegido. En el contraste entre el proyecto construido y el modelo deseado se constata que hay una desemejanza aún mayor que la semejanza. Si la semejanza que une la obra con el modelo produce satisfacción y la esperanza de una realización completa, la desemejanza, verificada por la experiencia, demuestra la imposibilidad real de alcanzar los valores propuestos por el prototipo. Esta es una de las diferencias principales de la imitación moderna respecto a las pre-modernas: la conciencia de no alcanzar nunca la perfección del modelo elegido como ideal. Movido por el deseo, el arquitecto comenzará una nueva

\section{EL PAISAJE COMO REFERENTE EN LA} ARQUITECTURA CONTEMPORÁNEA.

\section{En la actualidad, uno de los modelos}

elegidos por la arquitectura, de entre la pluralidad que existen, es el paisaje, que se manifiesta como un ideal a alcanzar, ya que es capaz de significar y representar los valores en los que se ve reflejada la sociedad actual. El proyecto de arquitectura, al elegir como referente al paisaje, busca una consistencia e identidad que lo integra en un sistema estético, histórico y cultural que lo rinde contemporáneo. Esta circunstancia es la que provoca el deseo de elegirlo como modelo por los arquitectos contemporáneos.

El deseo de paisaje significa siempre algo diferente para cada cultura y, por ende, para cada arquitecto. No es una idea uniforme ni común a todo el mundo; existen tantos paisajes como personas. Por ello, el paisaje ha sido indagado y declinado de forma intensa y diversa durante los últimos años. La arquitectura de Alvaro Siza es la escucha del paisaje vernáculo. Para Tadao Ando, el paisaje es la encarnación visible de la naturaleza, que ya no es estática, perfecta y acabada como en la premodernidad nueva figura de hada con caracteres imperfectos. Un hada, casi humana, que enferma y empobrece, al contrario del hada perfecta e inmaculada, inalcanzable de la tradición de las fábulas en la que se la figura de un mimo que después de haber interpretado su papel escribe el guión de lo actuado, es decir, realiza una mimesis inversa que no imita nada. Y un poco antes, Oscar Wilde termina el libro La decadencia de la mentira (1889) con la célebre boutade "...también la Naturaleza externa imita al Arte", invirtiendo el célebre postulado aristotélico que entonces tenía dos mil doscientos años. Esta nuev imitación contemporánea, que comienza a partir de los últimos decenios del siglo diecinueve, ha sido teorizada por Javier Gomá en su Teoría General de la Imitación (2003). La libertad y la subjetividad
reciproca de esta novedosa imitación que propone Gomá no existía en las antiguas imitaciones pre-modernas. El producto de la imitación actual ya no es una copia, un objeto, sino un sujeto libre que no alcanza nunca al modelo elegido, pero que obtiene una esperanza en la acción del imitar. 
occidental, sino que es un elemento dinámico y vivo en progreso constante. El paisaje, para Frank Gehry, es el que produce la propia mano del hombre, aceptando sus contradicciones y controversias, lo humilde, lo vulgar. La configuración nunca terminada de los espacios que proyecta Zaha Hadid es otro buen ejemplo de lo que significa el paisaje como modelo en la arquitectura contemporánea. Los espacios que la arquitecta iraquí proyecta ponen al usuario en una situación análoga a la experiencia emocional suscitada por el paisaje, porque permite moverse libremente por ellos, concretando una aspiración a la libertad que constituye otra de las cuestiones centrales del paisaje. Ese ideal de libertad del paisaje es uno de los valores donde se reconoce el arquitecto contemporáneo cuando lo elige como modelo de imitación.

Los paisajes son ejemplos útiles para entender la ciudad, porque nunca están terminados en sí, ni son objetos de atención; Son lo que Koolhaas denomina "campos de potencial", que permiten, sostienen y generan nuevas condiciones. James Corner (2014) sostiene que la ciudad es un paisaje casi geológico, además de una ecología evolutiva de sistemas, flujos e interacciones. Para este co-autor del High Line de Nueva York, la ciudad es similar a un bosque: es un organismo que crece y cambia constantemente. Es un tejido que sostiene el movimiento, el acontecimiento, el programa y el cambio. La arquitectura del paisaje cultiva esas potencialidades con un arte de construir que empieza pero que nunca termina.

El paisaje se vuelve interesante cuando es entendido ecológica, operativa y culturalmente, cuando se han investigado sus valores y se toma la decisión de adoptarlo como modelo válido para unas circunstancias concretas. En ese caso, los arquitectos lo utilizan como material incorporando las diferentes cualidades de los paisajes de los lugares donde se insertan sus obras. Es el caso del Pabellón de la Meditación en Unazuki (Japón), proyectado por Enric Miralles, en el que el recorrido discurre por lugares donde la cubierta es el cielo y las estructuras arquitectónicas construyen junto con la vegetación un nuevo paisaje, estimulando la concentración o el pensamiento sobre la naturaleza. El entendimiento del paisaje. como elemento dinámico y vivo, lo dota de un especial significado que lo convierte en un sujeto anhelado e indagado para poder utilizarlo de referencia durante el proceso de proyecto

La imitación del paisaje se enmarca en un contexto cultural en el que siempre se imita. El hombre vive inmerso en un mundo de modelos. Somos ejemplos rodeados de ejemplos. La tarea del hombre es convertir ese hecho en asumible, haciéndolo de una forma racional, libre, ética y moral. El arquitecto también está dentro de un horizonte de referentes, uno de los cuales es el paisaje, elegido como modelo por sus peculiares características o principios estructurales que dotan de consistencia e identidad al proyecto, enmarcándolo en la contemporaneidad.

Cada tiempo tiene su música y una dialéctica propia. Cada sociedad son palabras y notas. Las civilizaciones han construido siempre sus edificios más bellos sobre necesidades, deseos y sensaciones que acaban reflejando el momento que viven. Cada época graba sus señas de identidad en su forma de hablar, en su música, en su arquitectura. La identidad de nuestra época es su arquitectura convertida en paisaje. $\mathbf{\Delta}$
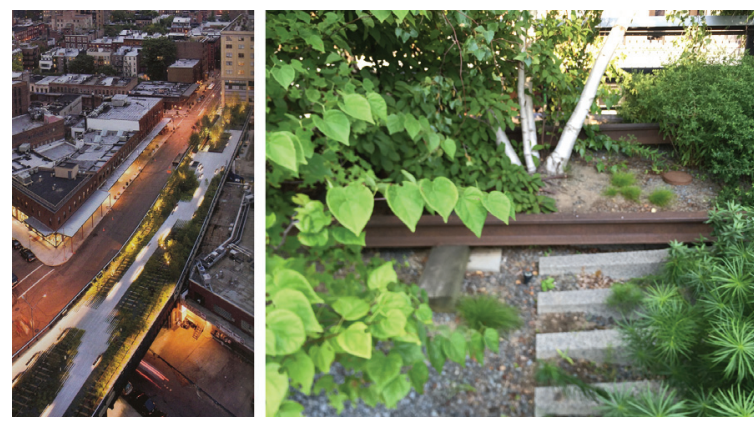

Imagen 5. High Line de Nueva York (USA), por Diller Scofidio + Renfro con James Corner Field Operations (2009) (fuentes: Izquiérda: Iwan Baan; Derecha: el autor 2015).

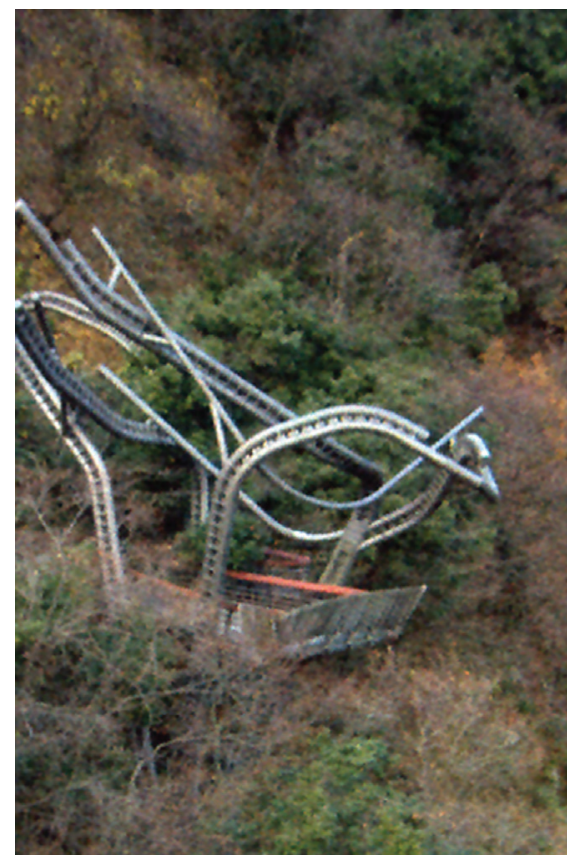
Imagen 6. Pabellón de la Meditación construido en 1993 en
Unazuki (Japón) por Enric Miralles (fuente: el autor 2001).

\section{REFERENCIAS}

AAVV 1987 Le Corbusier II viaggio in Toscana (1907) Venezia: Cataloghi Marsilio.

Ayala, F., 1996. "El paisaje y la invención de la realidad." Paisaje, juego y multilingüismo, Actas del X Simposic de la Sociedad Española de Literatura General y Comparada. Santiago de Compostela: D. Villanueva y F. Cabo (Ed.).

Borromini, F., 1993. Opus Architectonicum, Roma: Mario De Benedectis, De Rubeis ed.

Corner, J., 2014. The landscape imagination: collected essays of James Corner 1990-2010. Nueva York: Princeton Architectural Press.

Frampton. K..1991. "In search of the Modern Landscape". Denatured Visions. Landscape and Culture in the Twentieth Century. Nueva York: MoMA
Gomá, J. 2003. Imitación y experiencia. Valencia: Pre-textos.

Guerra, A., Morresi, M., 1996. Gabetti e Isola. Opere di Architettura. Milano: Electa. Howard, P., 2014. An introduction to landscape. Aldershot: Ashgate Jackson, J., 2010. Descubriendo el paisaje autóctono Madrid: Biblioteca Nueva Kaufmann, R., 1962. Frank Lloyd Wright: sus ideas y realizaciones. Buenos Aires: Victor Leru. Pedio, R., 1973. "Residenziale ovest a Ivrea." L'architettura: cronache e storia, 83: 212-213. Quesada-García, S., 2000. "Industria, Arquitectura, Paisaje: las Minas de Oro de Rodalquilar." Actas Segundo Seminario Docomomo lbérico. Barcelona: Docomomo lbérico, 217-223. 